\title{
Podoplanin neutralization improves cardiac remodeling and function after myocardial infarction
}

\author{
Maria Cimini, Venkata Naga Srikanth Garikipati, Claudio de Lucia, Zhongjian Cheng, Chunlin Wang, \\ May M. Truongcao, Anna Maria Lucchese, Rajika Roy, Cindy Benedict, David A. Goukassian, \\ Walter J. Koch, and Raj Kishore \\ Center for Translational Medicine, Department of Pharmacology, Lewis Katz School of Medicine, Temple University, \\ Philadelphia, Pennsylvania, USA.
}

\begin{abstract}
Podoplanin (PDPN), a small mucin-type transmembrane glycoprotein, has been recently shown to be expressed by lymphangiogenic, fibrogenic, and mesenchymal progenitor cells in the acutely and chronically infarcted myocardium. PDPN binds to a C-type lectin-like receptor 2 highly expressed by CD11 ${ }^{\text {hi }}$ cells following inflammatory stimuli. Why PDPN expression appears only after organ injury is currently unknown. Here, we characterize the role of PDPN in different stages of myocardial repair after infarction and propose a PDPN-mediated mechanism in the resolution of post-myocardial infarction (MI) inflammatory response and cardiac repair. Neutralization of PDPN led to significant improvements in the left ventricular (LV) functions and scar composition in animals treated with PDPN-neutralizing antibody. The inhibition of the interaction between PDPN and C-type lectin-like receptor 2 expressing immune cells in the heart enhances the cardiac performance, regeneration, and angiogenesis after MI. Our data indicate that modulating the interaction between PDPNpositive cells with the immune cells after MI positively affects immune cell recruitment and may represent a novel therapeutic target to augment post-MI cardiac repair, regeneration, and function.
\end{abstract}

Conflict of interest: The authors have declared that no conflict of interest exists.

Copyright: (c) 2019 American Society for Clinical Investigation

Submitted: December 20, 2018

Accepted: June 27, 2019

Published: August 8, 2019.

Reference information: /CI Insight. 2019;4(15):e126967. https://doi. org/10.1172/ji.insight.126967.

\section{Introduction}

The role of inflammation in the setting of myocardial infarction (MI) has been extensively studied, and these observations generated significant enthusiasm regarding the potential use of antiinflammatory strategies to reduce infarct size and to attenuate injury after MI (1). Therapeutic approaches have not yet succeeded, however, because impaired inflammatory cell recruitment that debride the wound, may negatively affect the scar formation and healing in the injured heart (2). Thus, new insights into understanding the post-MI modulation of inflammatory response is needed for the development of successful cardioprotective therapies. In this study, we investigated a potentially novel cell-cell interaction between PDPN-positive cells and inflammatory cells in the modulation of inflammation after MI. Characterization of PDPN expression after MI has recently been described suggesting PDPN expression by heterogenous populations of lymphangiogenic, fibrogenic, and mesenchymal progenitor cells (3).

PDPN is a mucin-like transmembrane glycoprotein that plays an important role in organ development, cell motility, tumorigenesis, and metastasis (4). Given its pleiotropic functions, PDPN is expressed by a wide variety of cells (5) including lymphatic endothelial cells, podocytes, alveolar type I cells, tumor cells, and cancer-associated fibroblasts (6). Importantly, PDPN appears in the infarcted heart as early as 2 days after MI (3). Lately, it has been documented that interstitial stromal cells acquire PDPN after organ injury (7) and under several pathological conditions such as fibrosis $(8,9)$ and autoimmune disease $(10,11)$. The expression of PDPN increases during inflammation in sepsis (12) and in animal models of deep vein thrombosis (13) and aortic calcification (14). In the adult heart, PDPN-positive cells are rare, constituting less than $5 \%$ of the myocardial small cell population (15). In fact, in homeostatic conditions PDPN is expressed only by cardiac lymphatic endothelial cells (3). In homeostatic conditions, PDPN is expressed only by cardiac lymphatic endothelial cells (3). Cardiac PDPN-expressing cells have been previously characterized by Cimini et al. (3), where they analyzed spatial and temporal distribution of the cells that acquire this glycoprotein after ischemic injury. Although PDPN is a common lymphoendothelial marker, it is expressed 
with an unexpected heterogeneity, and the appearance of PDPN-positive cells increases over time from the acute ( 2 days) to the chronic phase of the MI (2 weeks and 1 month) (3). The interstitial PDPN-positive cells did not express lymphatic vessel endothelial hyaluronan receptor 1 (LYVE-1), a specific lymphatic endothelial marker, prospero-related homeobox 1, a major transcription factor of the lymphatic endothelial fate, and VEGFR-3 unless organized in cardiac lymphatic vessels (16). This finding suggests that a large portion of PDPN-positive cells do not possess a differentiated lymphatic endothelial phenotype. Additionally, PDPN-positive cells do not express markers of mature endothelial cells like cluster of differentiation 31 (CD31) and VEGFR-2 (17). The immunohistochemistry and the flow cytometry analysis of the infarcted hearts at different time points after MI showed that the PDPN-positive cells were distinctly PDGFR- $\alpha$ positive. The colocalization of PDGFR- $\beta$ with PDPN was infrequent early after MI and strongly elevated at later stages of infarct healing in the mature scar. Because PDGFR- $\alpha$ is associated with the properties of immature mesenchymal cells and PDGFR- $\beta$ is a marker of pericytes, the concordance of costaining with PDPN suggested that PDPN-expressing cells also contain a population with progenitor capabilities. Of note, although PDGFR- $\alpha$ and PDGFR- $\beta$ are also associated with fibrogenic behavior, PDPN-positive cells do not express vimentin and $\alpha$-smooth muscle actin ( $\alpha$-SMA) at any time point, suggesting that PDPN-positive cells do not generate fully differentiated fibroblasts (3). PDPN therefore may represent a sign of activation of a cohort of cells during different phases of postischemic myocardial wound repair.

PDPN binds to C-type lectin-like receptor 2 (CLEC-2) $(18,19)$ that is exclusively expressed by platelets and a variety of immune cells (20). Interestingly, CLEC-2 is upregulated in CD11 b $\mathrm{b}^{\text {hi }}$ cells such as monocytes and macrophages following inflammatory stimuli (21). Under physiological conditions, PDPN in normal tissues does not have access to CLEC-2 on inflammatory cells (21); however, during developmental stages $(6,20,22)$ or under pathological conditions $(4)$, they interact with each other and play various important physiological or pathophysiological roles (4). Although the function of PDPN in development $(4,6,22)$ and tumor metastasis $(23)$ is well documented, the role of PDPN remains unclear in the repair of injured organs (7) and, in particular, in repair of the ischemic heart (3).

Here, we demonstrate that the neutralization of PDPN using neutralizing antibodies (Abs) improved heart function and scar composition after MI. In particular, we show that the interaction of PDPN-positive cells with monocytes and macrophages alters inflammatory responses in the phase of granulation after MI and leads to cardiomyocyte survival and/or regeneration in the infarct region. Our data support a potentially novel and alternative mechanism of interactome in the heart that, when neutralized, leads to altered inflammatory response and preservation of cardiac function and structure. Collectively, our data indicate that the neutralization of PDPN/CLEC-2 interaction after MI may represent a novel therapeutic strategy to augment cardiac repair and function after MI.

\section{Results}

Cardiac injury induces the expression of PDPN. PDPN expression has been reported to increase from the acute to the chronic phase of MI (3). Analysis of PDPN expression revealed that compared with sham-operated hearts, where PDPN was expressed only by lymphatic endothelial cells (Figure 1, A and B, labeled in red), hearts after MI showed a significantly augmented expression of PDPN in the infarct border zone (BZ) as early as 2 days after MI (Figure 1, C and D, labeled in red). Because different models of cardiac stress such as angiotensin II (Ang II) lead to cardiac fibrosis $(24,25)$, we also investigated PDPN expression in postAng II infusion-induced cardiac fibrosis. The fibrotic areas (Supplemental Figure 1A; supplemental material available online with this article; https://doi.org/10.1172/jci.insight.126967DS1) were positive for PDPN (Supplemental Figure 1, B and C, labeled in red) and negative for $\alpha$-SMA (Supplemental Figure 1B, labeled in green), suggesting that PDPN-positive cells are distinct from activated myofibroblasts and may play a distinct role in fibrosis regulation. Interestingly, PDPN was also highly expressed in the fibrotic cardiac tissue samples of human ischemic cardiomyopathy patients (Figure 1, E and F, showing the same area of interest, as well as Supplemental Figure 1D and Supplemental Figure 1E), suggesting that enhanced PDPN expression is not limited to failing murine hearts and that PDPN expression increases in fibrotic human diseased hearts.

To understand the biology of MI-induced PDPN-positive cells in detail, we isolated PDPN-positive cells from infarcted mouse hearts at 2 days after MI using magnetic beads, and the cells were culture expanded in vitro (Figure 2, A and B). We verified the purity of the cultured cells by flow cytometry, which showed a highly enriched population with minimal contamination with LYVE-1-positive lymphatic endothelial cells (Figure 2B). To confirm the cardiac source of these cells and rule out bone marrow (BM)/circulation source, 

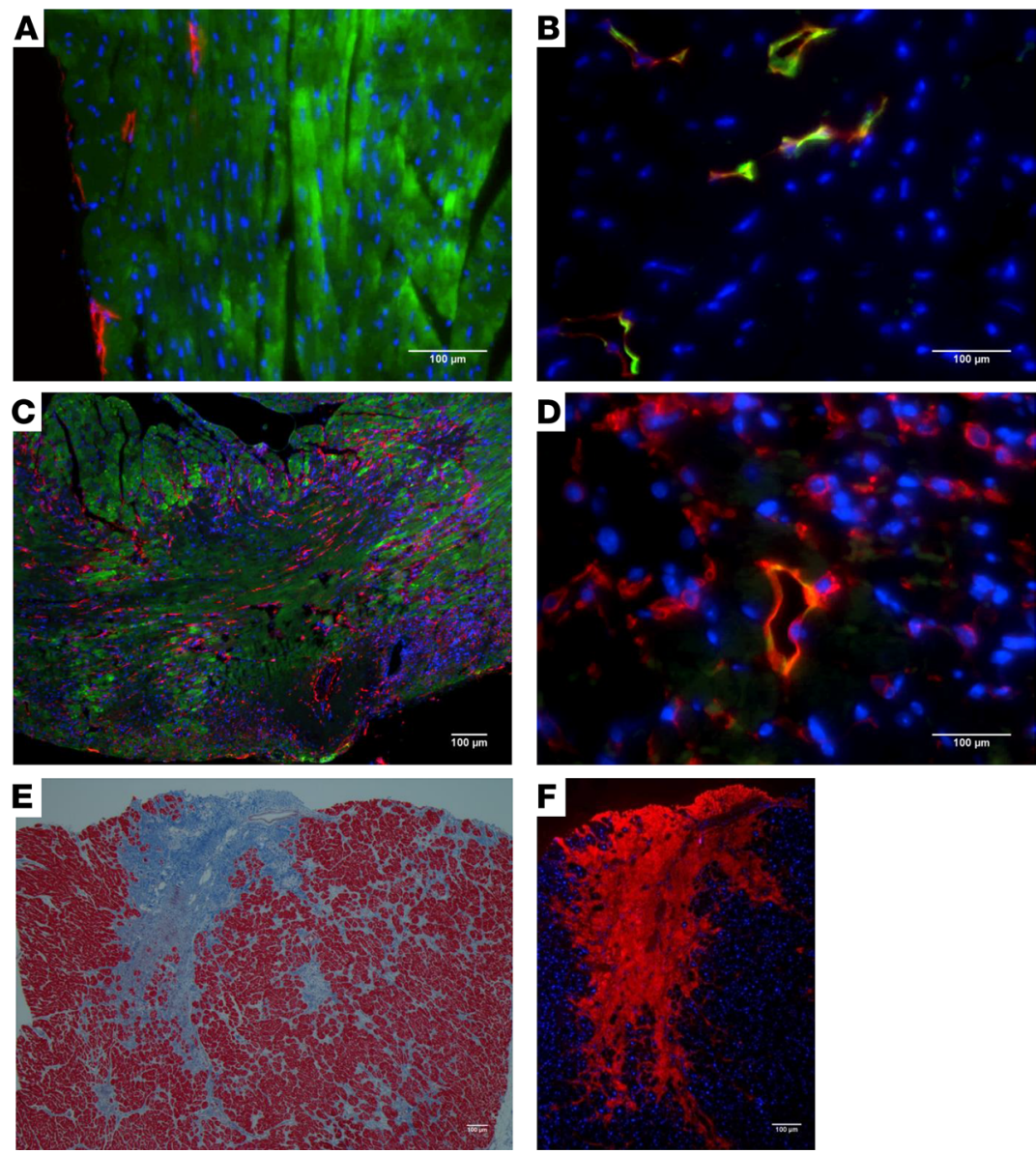

Figure 1. PDPN expression in mouse model of $\mathrm{MI}$ and human ischemic hearts. Thin cardiac sections from control sham-operated ( $\mathbf{A}$ and $\mathbf{B}$ ) and infarcted ( $\mathbf{C}$ and $\mathbf{D})$ mouse hearts at 2 days after surgery were indirectly immunolabeled with (A and $\mathbf{C}$ ) PDPN in red, $\alpha$-SARC actin in green, nuclei in blue, (B and $\mathbf{D})$ PDPN in red, LYVE-1 in green, and nuclei in blue, respectively. (C and $\mathbf{D}$ ) The area represents the infarct/BZ of the $\mathrm{Ml}$ and shows the increase in PDPN expression at 2 days after MI ( $n=3$ /group). ( $\mathbf{E}$ and $\mathbf{F}$ ) Thin cardiac sections obtained from ischemic human hearts were stained with Masson's trichrome (E) and (F) indirectly immunolabeled with PDPN Ab in red, nuclei in blue. Note that the infarcted/ fibrotic tissue in blue (E) corresponds to the area in red (F) and is highly positive for PDPN ( $n=5$ /group).

we additionally determined PDPN-expressing cells in the circulation of sham and post-MI mice. As shown by the scatter plots (Figure 2, C and D), none of the BM lineage-positive cells expressed PDPN, and this expression did not increase after MI, suggesting that PDPN-positive cells reside in the heart and are not recruited from the BM. To test the hypothesis that PDPN is a marker of injured myocardium and that blocking PDPN activity may lead to positive remodeling after MI, we first tested the neutralizing activity of anti-PDPN Abs to inhibit migration of PDPN-positive cells under inflammatory conditions by in vitro migration assays. PDPN-positive cells displayed an intense migratory capacity toward LPS-conditioned medium. This migration was inhibited by 2 independent PDPN-neutralizing Abs (Abs A and B, respectively), as shown in Figure $2 \mathrm{E}$. Both Abs showed similar inhibitory activity and were used for further in vitro and in vivo experiments.

PDPN neutralization improves cardiac function after MI. We next tested whether PDPN neutralization in vivo would enhance cardiac functions after MI. Mice were subjected to MI by permanent left anterior descending artery (LAD) ligation and were treated with 25- $\mu$ g i.p. injections of neutralizing Ab, $25 \mu \mathrm{g}$ of isotype-matched rabbit IgG, or saline solution at 1, 2, 7, and 15 days after MI. LV functions were evaluated in all mice at baseline and on days 7 and 30 after MI by echocardiographic measurements. These studies revealed that on day 7 after MI, LV ejection fraction (\% EF) (Figure 3A) and fractional shortening ( $\%$ FS) (Figure 3B) were significantly reduced in all the groups, while LV diameters at end-diastole and -systole (Figure 3, C and D) were equally increased, suggesting comparative infarct in treated and control (IgG and saline) groups. In contrast, 30 days after MI, the echocardiography data showed that PDPN neutralization 

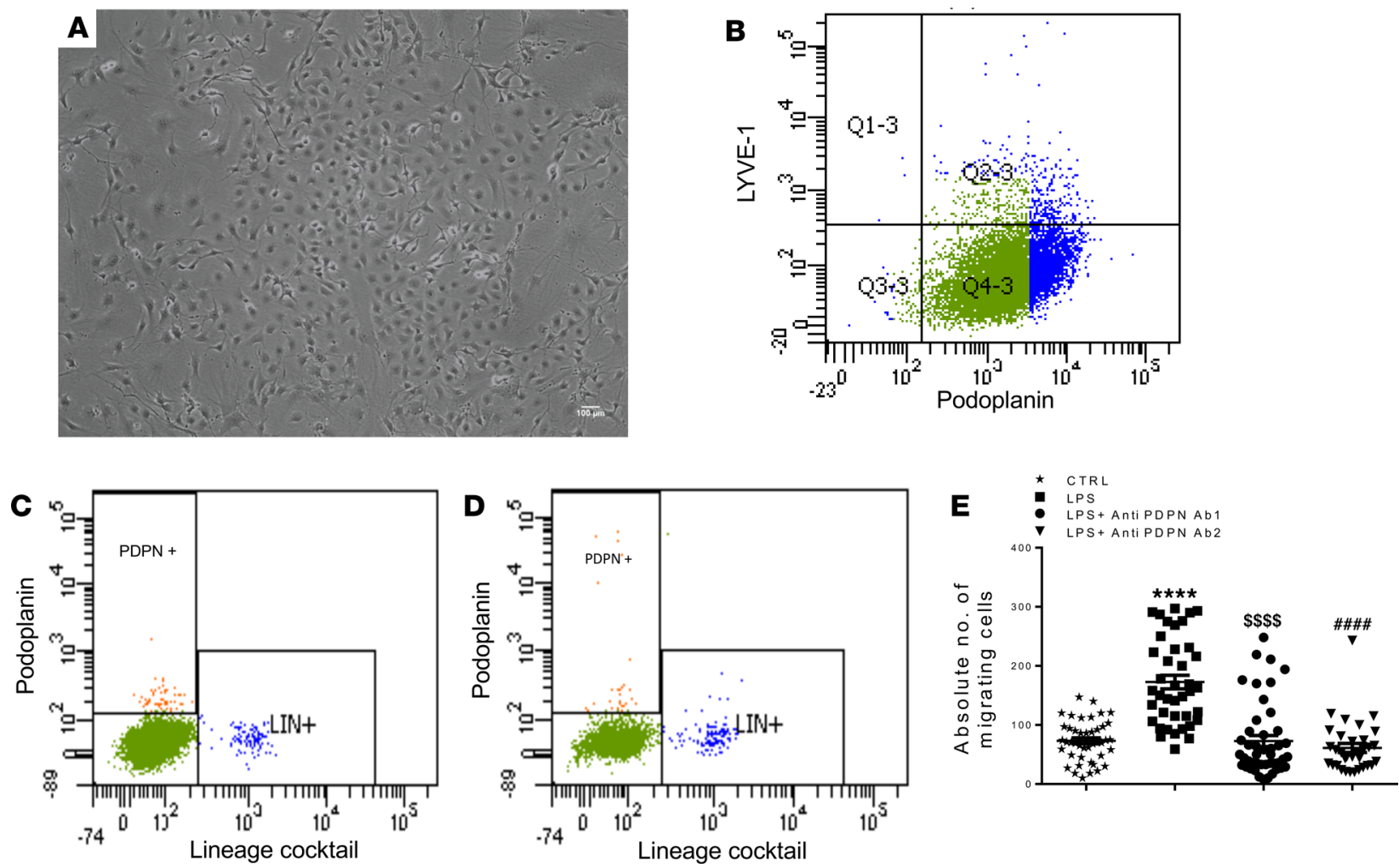

Figure 2. In vitro characterization of PDPN-positive cells isolated from infarcted mouse hearts. (A) Representative image of PDPN-positive cells in culture. (B) The PDPN-positive cells were isolated from infarcted mouse hearts 2 days after MI and culture expanded in vitro. The representative flow cytometry scatter plots of the flow cytometry analysis showed that $85 \%$ of the cell population is PDPN positive and LYVE-1 negative ( $n=3 /$ isolations). (C and D) Flow cytometry analysis of the circulating PDPN-positive cells in the peripheral blood of control (C, sham operated) and 2 days after MI (D) animals. Importantly, the BM lineage-positive cells (stained with the lineage-positive cocktail antibodies) do not express PDPN and the PDPN-positive cells did not increase in the circulation after myocardial infarction, $n=3$ /isolations. (E) Quantitative analysis of the PDPN-positive cells that migrated from the basal to the apical side of transwell inserts under different conditions; the migratory capacity of PDPN-positive cells toward LPS-conditioned medium was significantly neutralized by $5 \mu \mathrm{g} / \mathrm{mL}$ of anti-PDPN antibodies. Data are presented as mean \pm SEM. ${ }^{* * *} P<0.0001$ control versus LPS-conditioned medium. $\$ \$ \$ P<0.0001$ LPS-conditioned medium versus LPS-conditioned medium plus anti-PDPN Ab 1. \#\#\#\# $P<0.0001$ LPS-conditioned medium versus LPS-conditioned medium plus anti-PDPN Ab 2. One-way ANOVA and Bonferroni's post hoc test were performed among all groups. Data are represented as a mean value of 3 independent experiments. For each experiment, between 3 and 6 samples per group were used.

significantly improved LV functions compared with both groups of control animals (Figure 3, A-D). In addition to echocardiography, we also performed hemodynamic evaluations (Supplemental Data, Supplemental Figure 2, A-C). Animals treated with neutralizing Ab exhibited an improved LV contractility and relaxation in response to $\beta$-adrenergic stimulation with isoproterenol at 30 days after MI compared with the 2 groups of controls. Together, these data demonstrated significantly improved cardiac function after MI in the animals treated with PDPN-neutralizing Ab and suggested that neutralization of PDPN may be a new strategy for postinfarct structural and functional repair.

Selective inhibition of PDPN improves postinfarction remodeling. Improvements in cardiac function by PDPN neutralization were corroborated by cardiac histological assessments. Masson's trichrome staining of cardiac tissue sections obtained from saline-treated (Figure 4A, left panel) IgG control (Figure 4A, middle panel) and treated (Figure 4A, right panel) hearts at 30 days after MI showed a significant difference in the scar size among the 3 groups. In control animals (Supplemental Figure 3A, left and middle panel), the scar was extended through the LV wall, while in the treated animals, the scar was confined around the coronary artery ligation (Supplemental Figure 3A, right panel), and fibrosis infiltrated only in the BZ of the MI. Quantification of the infarct scar area confirmed that PDPN neutralization attenuated the adverse remodeling after MI (Figure 4A, graph). To further confirm the cardioprotective activity of the PDPN-neutralizing Ab, we performed TUNEL assay on treated and saline control mouse hearts at 3 days after MI; PDPN neutralization significantly reduced the number of TUNEL-positive cells (Supplemental Figure 3B). Scar tissue of treated animals displayed robust neovascularization of the healed tissue compared with control samples (Figure 4B, CD31 labeled in red). Addition- 

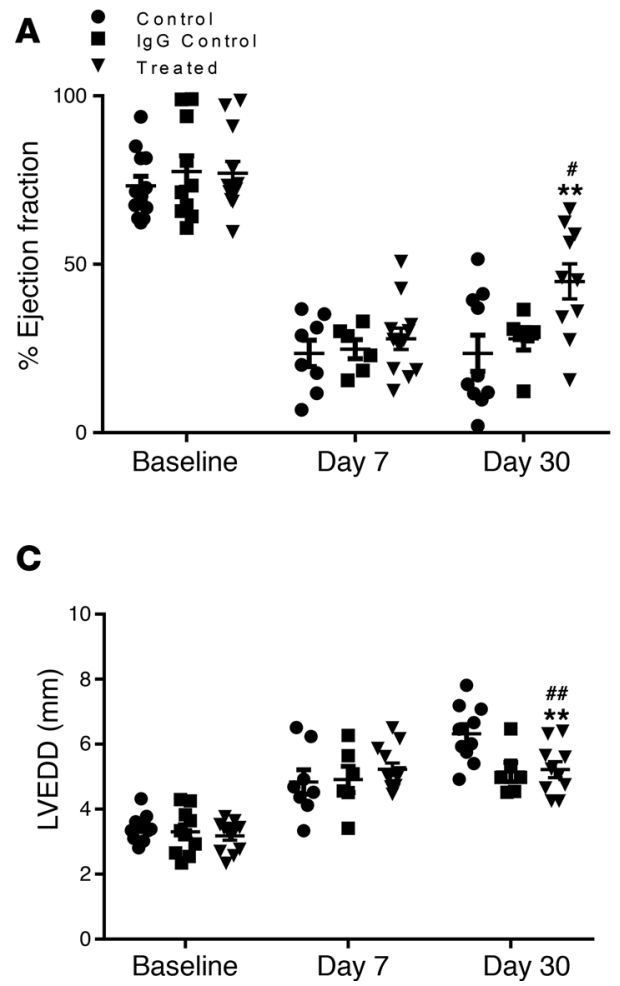

B

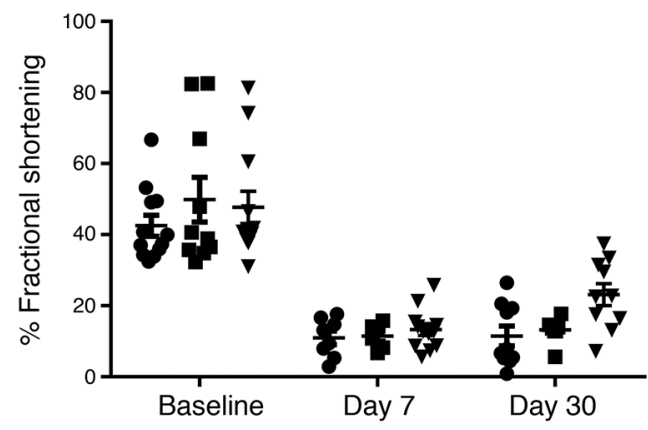

D

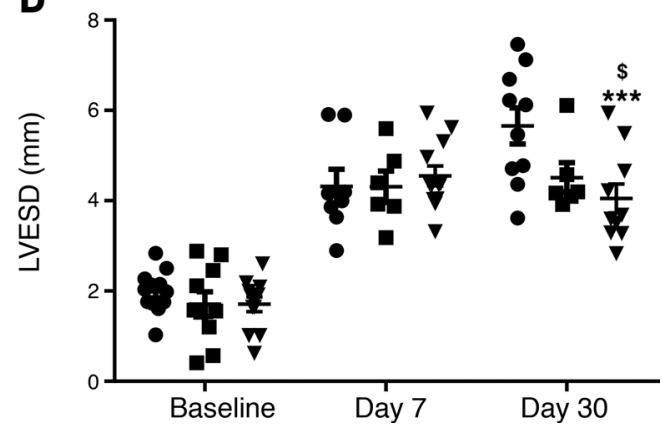

Figure 3. PDPN neutralization improves LV function after MI. Scatter plots representing EF percentage (A), percentage of FS (B), LV end-diastolic diameter (C), and LV end-systole diameter (D) as measured by echocardiography at baseline and at 7 and 30 days after MI in mice treated with PDPN-neutralizing Ab ( $n=12$ /group), isotype-matched IgG ( $n=10$ / group), or saline ( $n=12$ /group). Data are presented as mean \pm SEM. ${ }^{*} P<0.05,{ }^{*} P<0.01,{ }^{* * *} P<0.001$, control versus treated at 30 days after MI. ${ }^{\#} P<0.05,{ }^{\# \# P}<0.01$, IgG control versus treated at 30 days after Ml. ${ }^{\$} P<0.05$ control versus IgG control at 30 days after MI. Two-way ANOVA and Bonferroni's post hoc test were performed among all groups.

ally, myofibroblast proliferation and the $\alpha$-SMA expression were strongly inhibited in treated animals (Figure 4B, $\alpha$-SMA labeled in green) at 30 days after MI compared with control animals. The untreated animals were characterized by fibrotic scar expressing $\alpha$-SMA, and myofibroblasts were located lengthwise along the scar with fibronectin assemblies (Figure 4C, fibronectin labeled in green) of the extracellular matrix. In contrast, in treated animals, fibronectin was organized in structures similar to swirls (Figure 4C, fibronectin labeled in green), which suggest further new vessel formation. Of note, new lymphangiogenesis (Figure 4C, LYVE-1 labeled in red) was not inhibited by the PDPN-neutralizing $\mathrm{Ab}$ in the treated animals where regular new lymphatic vessel formation was observed (Figure 4C), confirming that the lymphatic endothelial cells do not represent an off-target PDPN-expressing cell population.

PDPN neutralization stimulates endogenous tissue regeneration after MI. Neovascularization and organization of the scar fibrosis are important for the recruitment and proliferation of progenitor cells involved in tissue repair (26). Differentiated cells could be derivative of resident progenitor cells in the tissue or recruited from the circulation; therefore, we investigated the presence of undifferentiated cells at the time of tissue granulation. Interestingly, we found a significantly higher number of immature NKX-2.5-expressing cells in the infarcted tissue of treated animals compared with controls at 7 days after MI (Figure 5A, NKX-2.5 labeled in red). We further identified groups of small nucleated cells in niches that were double positive for NKX-2.5 and troponin T (Figure 5B, NKX-2.5 labeled in red and troponin T labeled in green), suggesting these cells may represent undifferentiated cardiac progenitors that may potentially differentiate into the myocyte lineage. These double-positive cells either in cluster (Figure 5B) or as isolated single cells (Supplemental Figure 4C) were found dispersed within the scar or in multiple areas of the BZ of the ischemic tissue in all heart samples of animals treated with PDPN-neutralizing Ab (Supplemental Figure 4B). Few NKX-2.5-positive cells were also identified in the control animals (Figure 5, A and B); none of which was positive for troponin $\mathrm{T}$ (Figure 5B). Interestingly, we documented organized groups of small myocytes within the scar tissue of animals treated with PDPN-neutralizing Ab at 30 days after MI (Figure 

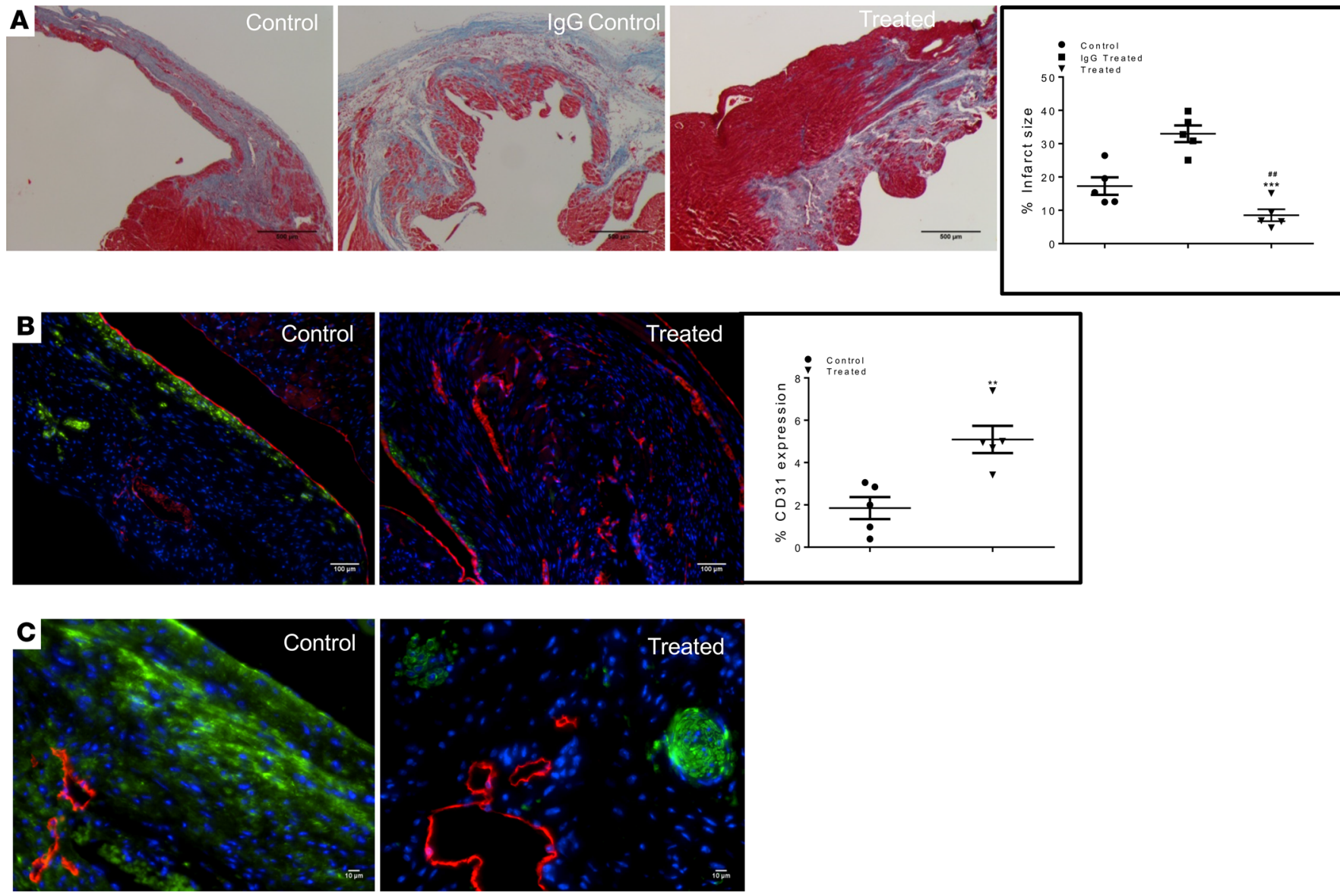

Figure 4. PDPN neutralization reduces infarct size and cell death and stimulates angiogenesis after MI. (A) Thin cardiac sections of saline treatment (left panel), IgG control (middle panel), and PDPN-neutralizing Ab-treated (right panel) mouse hearts at 30 days after MI were labeled with Masson's trichrome staining. Treated animals (A, right panel) displayed a smaller scar compared with the animals in both control groups (A, left and middle panels). Quantitative analysis of the cardiac scar size showed significant reduction in the infarct area in the treated animals compared with the controls (graph, $\mathbf{A})(n=5 / g r o u p)$. Data are presented as mean \pm SEM. ${ }^{*} P<0.05 \mathrm{lg} G$ control versus treated and ${ }^{\#} P<0.05$ saline control versus treated. One-way ANOVA and Bonferroni's post hoc test were performed among all groups. (B) Thin cardiac sections of saline control (left panel) and PDPN-neutralizing Ab-treated (right panel) mouse hearts at 30 days after MI were immunolabeled with CD31 (red), $\alpha$-SMA (green), and nuclei (blue). The new vessel formation in the scar tissue of treated animals (B, right panel) was significantly enhanced compared with the controls (B, left panel), while $\alpha$-SMA positivity was reduced in the treated animals (B, right panel, graph). ${ }^{*} P<0.05$ control versus treated ( $n=5$ /group). Student's $t$ test was performed between the groups. (C) Thin cardiac sections of saline-treated control (left panel) and PDPN-neutralizing Ab-treated (right panel) hearts at 30 days after MI were indirectly immunolabeled with LYVE-1 (red), fibronectin (green), and nuclei (blue). PDPN neutralization did not affect neolymphangiogenesis and reduced the fibronectin distribution along the scar.

$5, \mathrm{C}-\mathrm{E})$. The small myocytes were characterized by expression of $\alpha$-sarcomeric actin ( $\alpha$-SARC) (labeled in green), expressed connexin 43 gap junction (Figure 5C, connexin 43 labeled in red), and showed functional contractile proteins troponin $\mathrm{T}$ (Figure 5D, troponin $\mathrm{T}$ labeled in red) and troponin I (Figure 5E, troponin I labeled in red). The figures show a consecutive section of the same area. A remote area of the same samples was used as an internal control (Supplemental Figure 4, E-G). These islands of small myocytes were rarely found in scars of control animals. These intriguing data suggest that events downstream of PDPN neutralization foster the progenitor cell survival likely by altering the scar microenvironment; therefore, we next investigated the interaction between PDPN and CLEC-2-expressing cells and how this interaction may regulate inflammatory and proliferative phases of the inflammation after MI.

PDPN neutralization alters inflammatory responses in injured myocardium. Based on the sequential migration of inflammatory cells after MI, we focused our attention on the monocyte/macrophage (27) populations as PDPN-interacting cells because monocyte recruitment to the heart follows a similar time frame as the appearance of PDPN (1). As previously described (21), we first verified that PDPN-positive cells (labeled in red) interact in vivo with CD68 (Figure 6A, CD68 labeled in green) and CD163-positive cells (Figure 6B, CD163 labeled in green) 2 days after MI using 2 complementary approaches of histological and flow cytometry analyses. The histological analysis of the immune infil- 

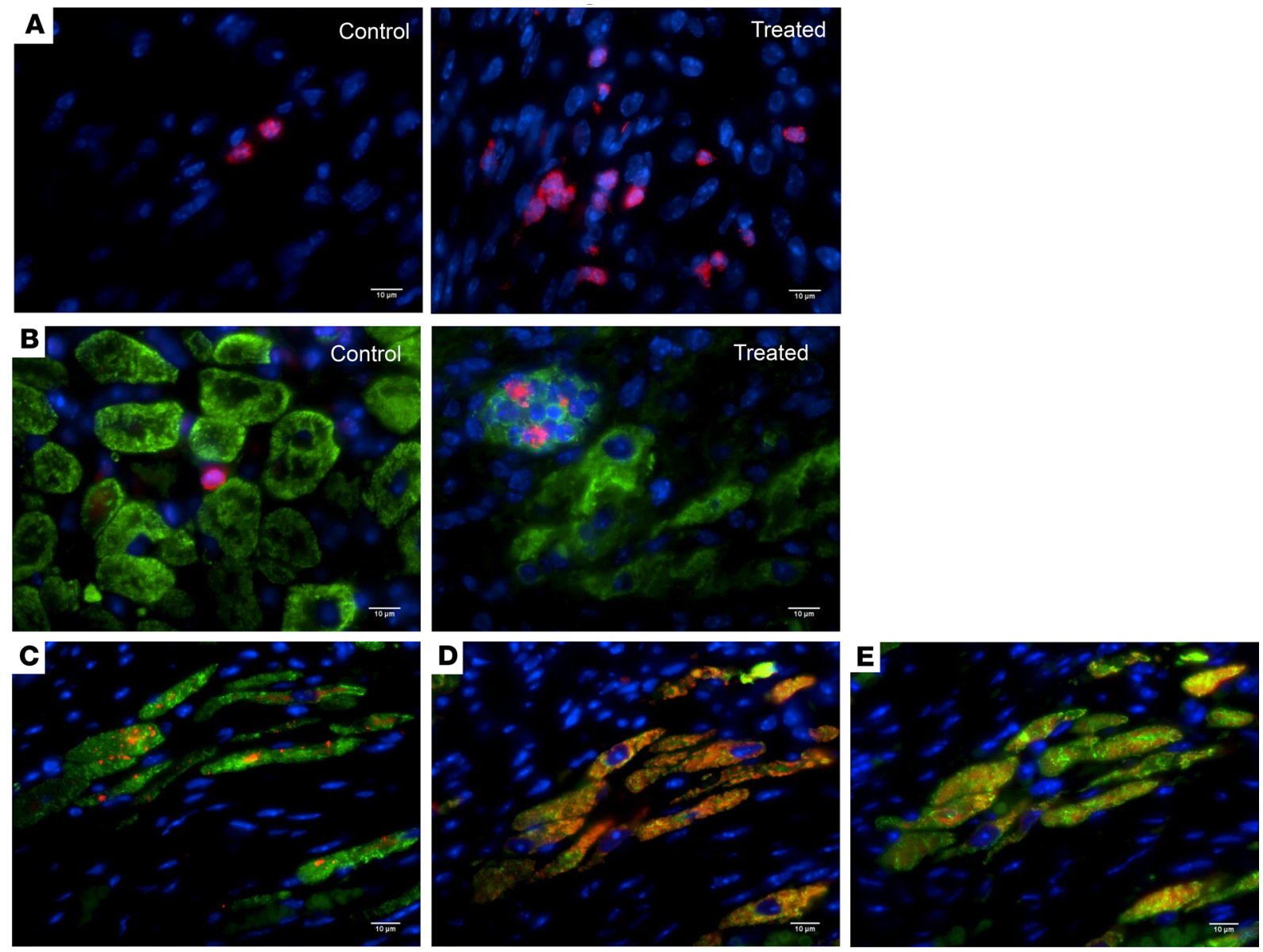

Figure 5. PDPN neutralization enhances endogenous tissue regeneration after MI. (A) Thin cardiac sections of saline control and PDPN-neutralizing Abtreated mouse hearts at 7 days after MI were indirectly immunolabeled with NKX-2.5 (red) and nuclei (blue). The ischemic heart tissue of animals treated with PDPN-neutralizing Ab was characterized by a significantly higher number of NKX-2.5-expressing cells (A, right panel) compared with the untreated group (A, left panel). (B) Thin cardiac sections of saline control and PDPN-neutralizing Ab-treated mouse hearts at 7 days after MI were indirectly immunolabeled with NKX-2.5 (red), troponin T (green), and nuclei (blue). The NKX-2.5-positive cells were found dispersed at the BZ of the MI or organized in groups of cells, resembling niches. All NKX-2.5-positive cells were also positive for troponin T (B, right panel). Some NKX-2.5-positive cells were also present in the scar/BZ of the untreated animals but none expressed troponin T (B, left panel) ( $n=3 /$ group). In order to correlate the NKX-2.5-positive small myocytes seen in the treated animals at 7 days after MI, we immunolabeled (C-E) thin cardiac sections of PDPN-neutralizing Ab-treated mouse hearts at 30 days after MI with connexin 43 (C) (red), troponin T (D) (red), troponin I (E) (red), $\alpha$-SARC actin (green) (C-E), and (nuclei) blue. The scar of animals treated with PDPN Ab (C-E) was characterized by islets of small myocytes expressing these myocyte markers (C-E) ( $n=5 /$ group).

trate at 3 days after $\mathrm{MI}$ in the control and treated groups showed 2 different immunological landscapes. Monocytes positive for CD68 (labeled in green) and CD163 (labeled in red) were present in the injured LV area of both groups of animals; in the control group, monocyte infiltration was predominately composed of $\mathrm{CD}^{+} 8^{+}$cells (Figure 6C) compared with the CD163 population (Figure 6C, graph). Animals treated with neutralizing $\mathrm{Ab}$, however, displayed a monocyte composition dominated by $\mathrm{CD} 163^{+}$ cells compared with the $\mathrm{CD} 68^{+}$population (Figure 6D). Histological data were further confirmed by flow cytometry analysis of mononuclear cells isolated by digestion of mouse hearts of treated and untreated animals at 3 days after MI. The single-cell suspension was stained for CD86, CD68, CD163, and CD206. The pan monocyte/macrophage marker CD86 showed similar cell numbers between control and treated animals (Figure 6E). However, the monocyte/macrophage population resident in the hearts of animals treated with PDPN-neutralizing Ab was predominantly positive for CD163 (Figure $6 \mathrm{G}$ ) and $\mathrm{CD} 206$ (Figure $6 \mathrm{H}$ ), whereas in the control group, it was predominantly positive for CD68 (Figure $6 \mathrm{~F}$ ). These data indicate that there was a marked recruitment or in situ differentiation of antiinflammatory monocytes/macrophages (CD163/CD206) in the infarcted myocardium of animals treated with PDPN-neutralizing $\mathrm{Ab}$; therefore, we next assessed cytokine and chemokine expression patterns to characterize the biosignals following the neutralization of PDPN-binding monocytes. 

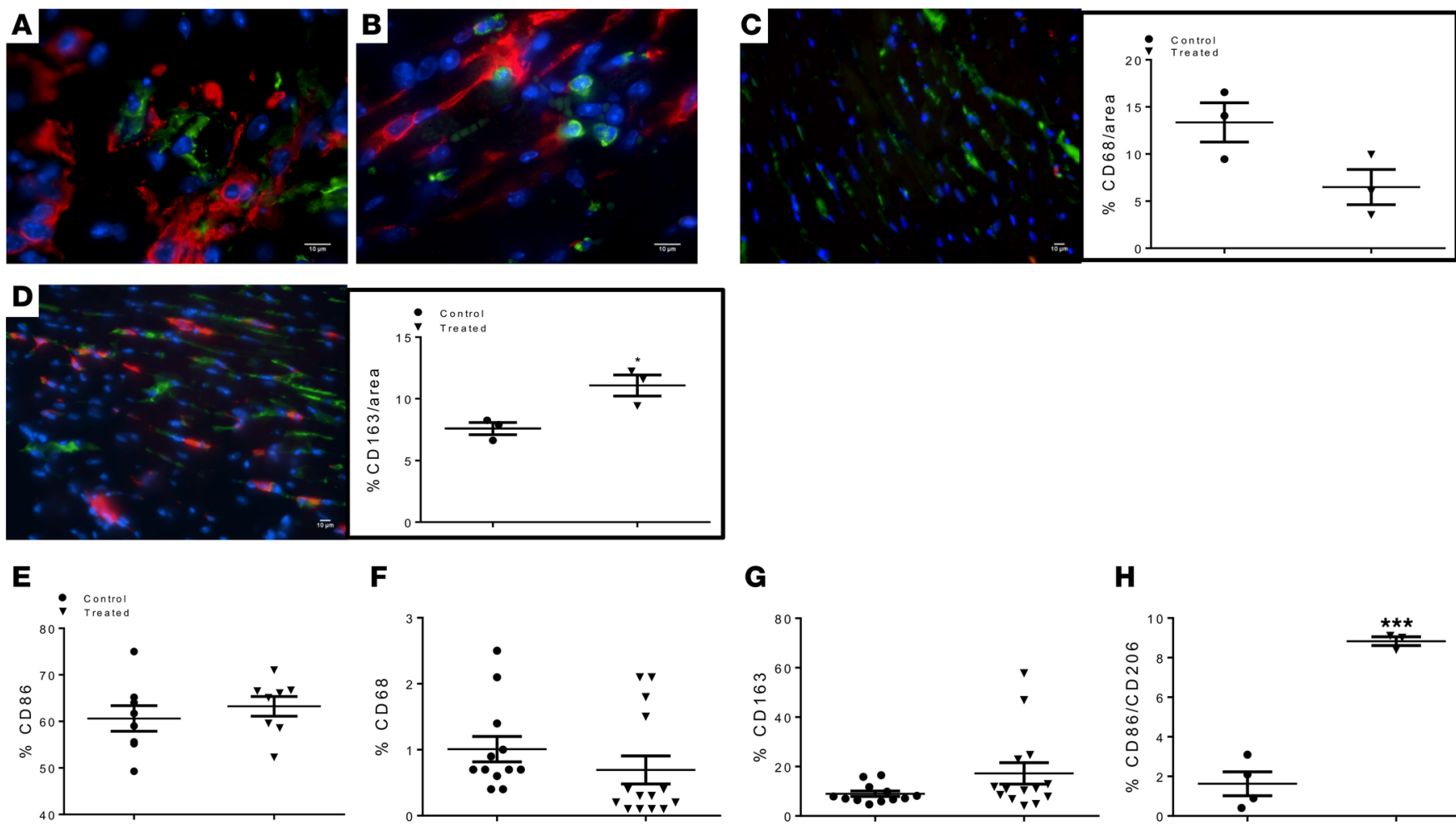

$\mathbf{F}$

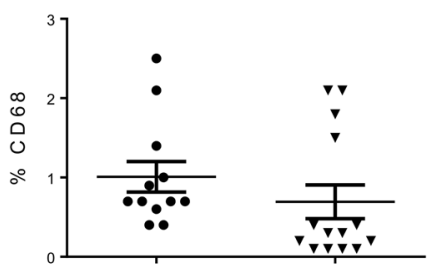

G

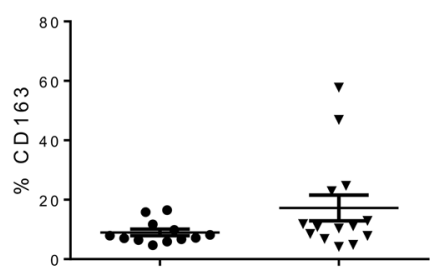

H

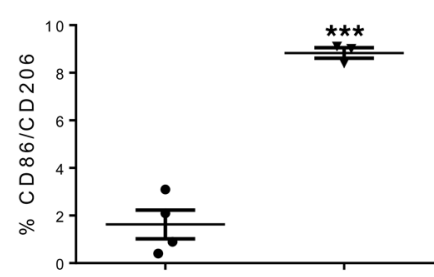

Figure 6. PDPN neutralization inhibits monocyte activation in vivo. (A and B). Thin cardiac sections from infarcted mice 3 days after MI were immunolabeled for PDPN (red) and CD68 (A), CD163 (B) (green), and nuclei (blue). PDPN-positive cells do not express CD68 and CD163, whereas CD68 ${ }^{+}$and CD163+ cells do not express PDPN. (C) Thin cardiac sections from saline control and (D) PDPN-neutralizing Ab-treated infarcted mouse hearts at 3 days after MI were indirectly immunolabeled with CD163 (red), CD68 (green), and nuclei (blue). Scars of treated animals (D) were characterized by a higher number of $\mathrm{CD}_{163}{ }^{+}$cells compared with the saline control animals where the majority of the monocyte/macrophage population expressed CD68 (C). Quantitative analysis of the distribution of $\operatorname{CD68}\left(\mathbf{C}\right.$, graph) and $\mathrm{CD}_{163^{+}}\left(\mathbf{D}\right.$, graph) cells was performed ( $n=3 /$ group). Data are presented as mean $\pm \operatorname{SEM}$. ${ }^{*} P<0.05$ saline control versus treated. Student's $t$ test was performed for analysis of differences between the groups (45). Flow cytometry analysis of the total resident monocyte/macrophage population isolated from the heart 3 days after MI confirmed the histology data. Mice treated with PDPN-neutralizing Ab were characterized by a higher percentage of $\mathrm{CD}_{163^{+}}(\mathbf{C})$ and $\mathrm{CD}_{206}(\mathbf{H})$ cells compared with the untreated animals where the monocyte/macrophage population was highly positive for CD68 (F). The total number of monocyte/macrophage recruited in the saline control and treated hearts was similar (E). Data are presented as mean \pm SEM. ${ }^{* *} P<0.001$ control versus treated ( $n=3-4$ /group). Student's $t$ test was performed between the groups.

To investigate whether neutralization of PDPN-positive cell binding to monocytes may lead to monocyte/macrophage phenotype switch, we isolated primary BM monocytes from C57BL/6 WT mice and activated them in vitro in the presence of LPS. Compared with naive monocytes, activated monocytes displayed an increased population of CD68-expressing cells as verified by flow cytometry (Figure 7B). LPS-activated monocytes were negative for CD163 (Figure 7C). Because LPS-stimulated monocytes mimic the in vivo activated and recruited monocyte population, we cocultured PDPN-positive cells with activated monocytes with and without the presence of PDPN-neutralizing Ab for 24 hours and determined CD68 expression by flow cytometry. Our results showed that LPS-treated monocytes remained activated when cocultured with PDPN-positive cells (Figure 7D). In contrast, PDPN-positive cells pretreated with PDPN-neutralizing Ab could not interact well with activated monocytes; the inhibition of the interaction reduced the expression of CD68 (Figure 7D) on activated monocytes. PDPN-positive cells did not express CD68 (Figure 7A). Furthermore, expression of selected cytokines and chemokines in the conditioned medium from PDPN-positive cells cocultured with LPS-activated monocytes with and without neutralizing $\mathrm{Ab}$ was assessed by quantitative ELISA. The cytokine and chemokine composition diverged accordingly with the 2 setups of coculture: proinflammatory IL-1 $\alpha$ (Figure 7E) and IL-12 (Figure 7F) were upregulated when monocytes bind PDPN-positive cells. In contrast, IL-9 (Figure 7G) and IL-10 (Figure 7H) were highly expressed in the presence of PDPN-neutralizing Ab. Our cytokine and chemokine analysis revealed a different pattern of inflammatory mediators in response to PDPN-positive cells binding to activated monocytes and a shift toward reparative monocyte/macrophage populations by the PDPN neutralization. 
A
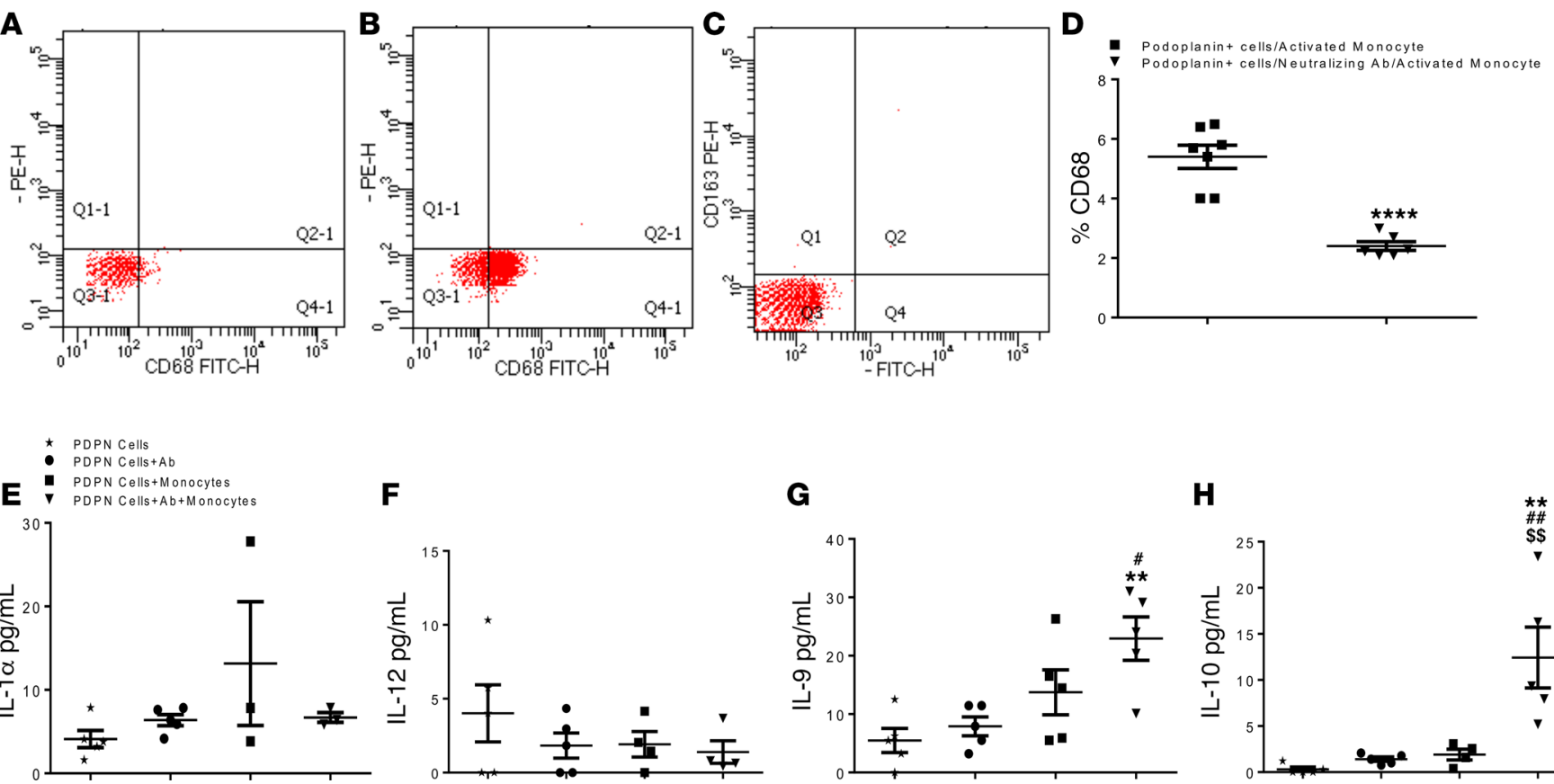

H
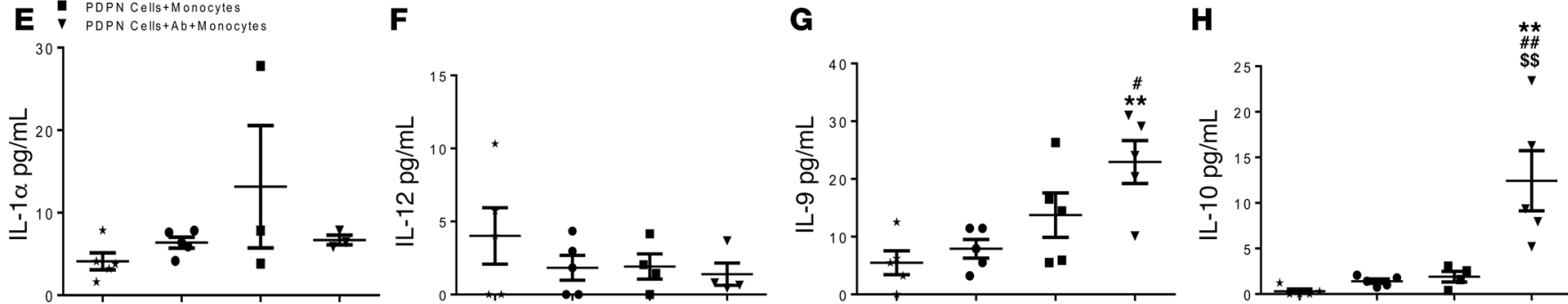

Figure 7. Podoplanin neutralization represses monocyte activation in vitro. Flow-cytometry analysis of cultured podoplanin positive cells and bone marrow isolated monocytes cultured in vitro and stimulated with LPS. (A) Podoplanin positive cells do not express CD68, (B) LPS activated monocyte highly expressed CD68 and were negative for CD163 (C). The flow-cytometry scatterplots are representive of biological triplicate isolations. (D) The graph represents the quantification of the flow-cytometry analysis of the monocytes CD68 expression after cocultured with podoplanin positive cells, with and without the presence of podoplanin neutralizing antibody. Data shows significant reduction of CD68 expression when podoplanin positive cells and LPS-stimulated monocyte were co-cultured in the presence of podoplanin neutralizing antibody. Data are presented as mean \pm SEM ${ }^{* * * *} P<0.01$ monocytes cocultured with podoplanin positive cells vs. monocytes co-cultured with podoplanin positive cells in the presence of podoplanin neutralizing Ab. Student's $t$-test was performed between the groups. $n=3$ /group, the data from biological triplicates in each separate experiment were consistent. Conditioned medium obtained from podoplanin (PDPN) positive cells only, podoplanin positive cells treated with neutralizing podoplanin antibody, podoplanin positive cells cocultured with activated monocytes with and without the presence of neutralizing podoplanin antibody were analyzed by ELISA for proinflammatory $(\mathbf{E}$ and $\mathbf{F})$ and antiinflammatory $(\mathbf{G}$ and $\mathbf{H})$ cytokines. The presence of the neutralizing antibody drastically reduced secretion of IL-1 $\alpha$ (E) and increased IL-10 secretion (H). Data are presented as mean $\pm \mathrm{SEM}{ }^{*} P<0.02$ podoplanin positive cells only vs. podoplanin positive cells co-cultured with activated monocytes with neutralizing antibody, ${ }^{\#} P<0.05$ and ${ }^{\# \#} P<0.02$ podoplanin positive cells with podoplanin neutralizing antibody vs. podoplanin positive cells cocultured with activated monocytes with neutralizing antibody, ${ }^{\$ \$} P<0.02$ podoplanin positive cells cocultured with activated monocytes without neutralizing antibody vs. podoplanin positive cells cocultured with activated monocytes with neutralizing antibody. $n=3-5 /$ group. One-way ANOVA analysis and Bonferroni post hoc test have been performed among all groups.

In order to further investigate the activation and the phenotype of the monocytes/macrophages that may or may not interact with PDPN- positive cells, we isolated PDPN-positive cells from a global GFPtransgenic mouse (C57BL/6-Tg [UBC-GFP] 30Scha/J) (Supplemental Figure 5, B and C) and cocultured PDPN-positive cells with LPS-activated monocytes isolated from the BM of WT C57BL/6J mice in a 1:5 ratio (Supplemental Figure 5A). The 2 cell types were cocultured with (Supplemental Figure 5C) and without (Supplemental Figure 5B) the presence of PDPN-neutralizing Ab. After 24 hours of coculture, the monocytes were separated from GFP-expressing PDPN cells by magnetic bead columns (Supplemental Figure 5A). For this experiment, we used GFP-expressing PDPN-positive cells because it has been reported that activated monocytes can acquire PDPN (28); thus, we preferred to separate the 2 types of cells using GFP as a surface marker. After the magnetic cell separation, the monocytes/macrophages were evaluated for the expression of major pro- and antiinflammatory cytokine transcripts by quantitative PCR (Supplemental Figure 5). Our data showed that the mRNA levels of IL-1 $\beta$ (Supplemental Figure 5D) and CD11b (Supplemental Figure 5J) were reduced in the monocytes cocultured in the presence of PDPN-neutralizing $\mathrm{Ab}$. At the same time, the mRNA levels of reparative M2 macrophage lineage markers such as arginase 1 (ARG-1), chitinase-3-like protein 1 (CHI3I3), and CD163 were increased (Supplemental Figure 5, F-H). The mRNA expression of CD68 (Supplemental Figure 5I) did not change within the groups, although we observed a reduction of the CD68 receptor by flow cytometry analysis (Figure 6D). The IL-10 mRNA level (Supplemental Figure 5E) did not change between the groups, although we observed a significant increase of secreted IL-10 protein by ELISA assessment (Figure 7H). This discrepancy between IL-10 mRNA and 

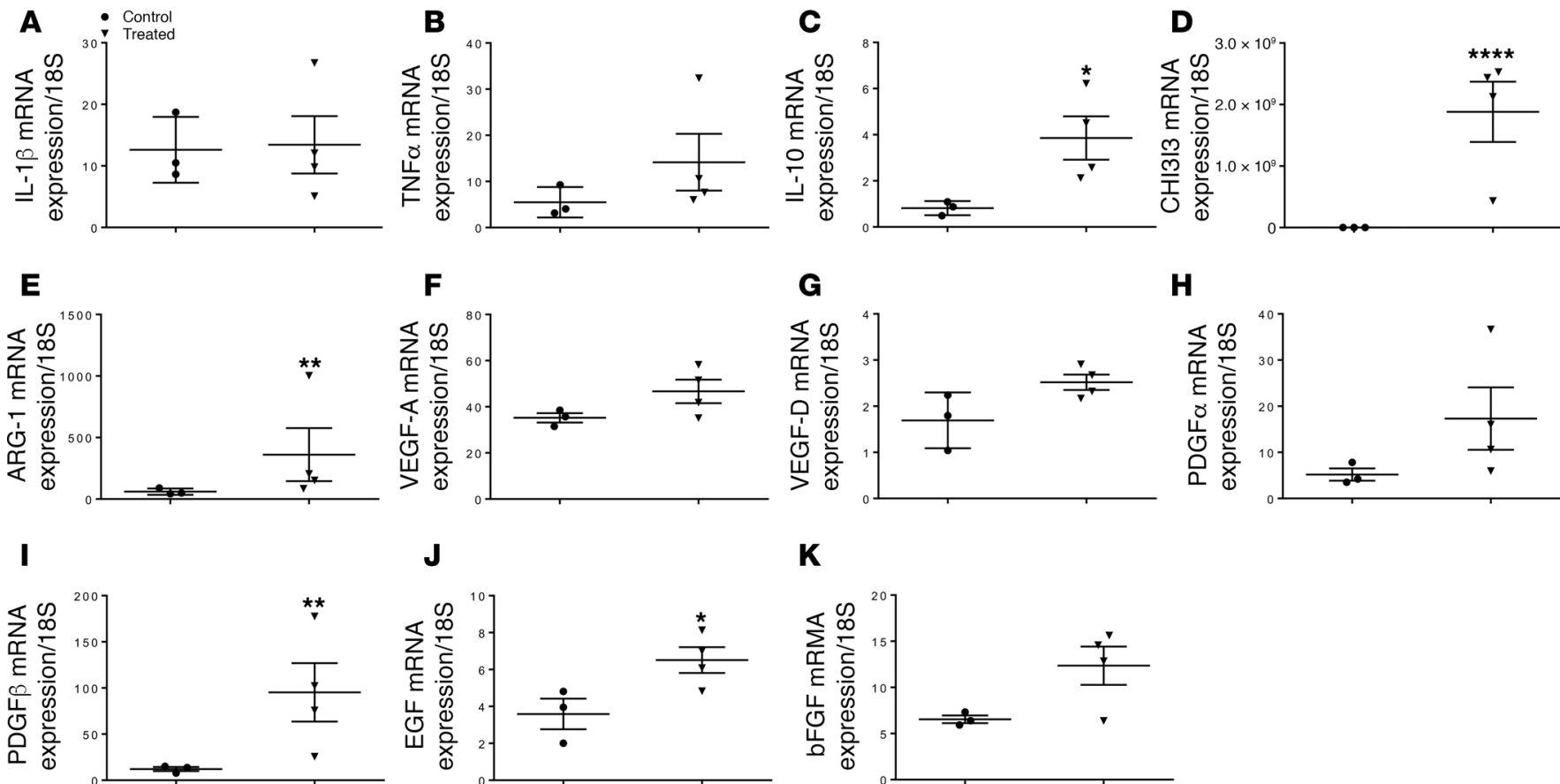

\section{$\mathbf{J}$}

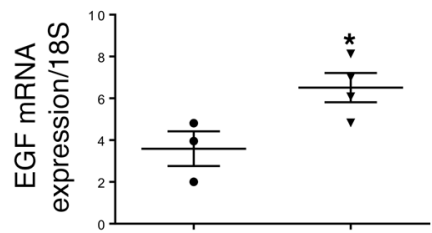

$\mathbf{K}$

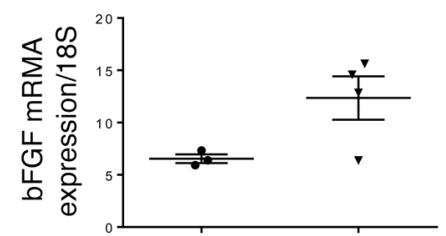

Figure 8. Podoplanin neutralization alters infarct microenvironment. Real time q-PCR analysis for the major proinflammatory (A and B) and antiinflammatory (C-E) cytokines and angiogenic growth factors (FK) was performed on cardiac tissues from podoplanin neutralizing antibody treated and untreated (saline) mice 3 days after myocardial infarction (MI). Quantitative analysis of IL-1 $\beta$ (A) and TNF $\alpha$ (B) mRNA indicated that podoplanin neutralizing antibody does not inhibit the gene expression these proinflammatory cytokines, but interestingly the antiinflammatory cytokine IL-10 (C), and the reparative monocytes markers: CHI3I3 (D) and ARG-1 (E) mRNA are significantly upregulated at day 3 post-infarct hearts. Neutralizing antibody treatment also led to an increased expression of the major growth factors for the tissue regeneration such as VEGF-A (F), VEGF-D (C), PDCF $\alpha(\mathbf{H})$, PDCF $\beta(\mathbf{I})$, ECF $(\mathbf{J})$ and bFGF $(\mathbf{K})$. Data are presented as mean \pm SEM ${ }^{*} P<$ $0.05,{ }^{* *} P<0.02$ and ${ }^{* * *} P<0.01$ saline control vs. treated. $n=3 /$ group. Student's $t$ test was performed between the groups.

protein may likely reflect a relative short half-life of IL-10 mRNA (29); thus, the analysis of the secreted IL-10 protein is much more accurate.

To gain additional insights into PDPN neutralization-mediated immunomodulation in vivo, we further investigated the pro- and antiinflammatory protein pattern to complement in vitro expression data. Expression analysis of selected chemokines was performed on tissue samples of mouse hearts after MI to investigate differences of the inflammatory mediators in animals treated with PDPN-neutralizing Ab compared with the untreated control group. In agreement with in vitro expression data, various cytokines were differentially expressed between the 2 groups: proinflammatory cytokines IL- $1 \beta$ and TNF- $\alpha$ (Figure 8 , A and B) were highly expressed in both groups, while antiinflammatory mediators such as IL-10, CHI3I3, and ARG-1 (Figure 8, C-E) were upregulated only in the treated group. The immunological landscape between treated and untreated animals diverged even further in the analysis of the growth factor profile. Limited expression of proangiogenic and proregenerative growth factors was observed in the untreated group. In accordance with the histological data of augmented vessel formation and progenitor cell proliferation in the treated group, quantitative real-time (qRT-PCR) analysis confirmed an upregulation of VEGF-A (Figure 8F), VEGF-D (Figure 8G), PDGFR- $\alpha$ (Figure 8H), PDGFR- $\beta$ (Figure 8I), EGF (Figure $8 \mathrm{~J}$ ), and basic fibroblast growth factor (Figure $8 \mathrm{~K}$ ) only in the treated group. The differences in cytokine and growth factor composition between treated and untreated animals suggested that PDPN neutralization led to a favorable microenvironment more conducive to endogenous repair.

\section{Discussion}

Exacerbated and prolonged inflammatory response is the leading cause of adverse remodeling after myocardial injury (30). Targeted antiinflammatory approaches were widely studied to reduce inflammation and improve cardiac repair (2), although patients treated with highly selective strategies did not show a positive outcome after therapy (2). The translational failure may be the result of exclusive inhibition of the 
recruitment of proinflammatory monocytes and decreased cytokine expression in the ischemic hearts (2). The complete suppression of the inflammatory pathways interferes with the migration and activation of reparative and regenerative cells important for positive tissue remodeling (2). In this study, we investigated an alternative pathway involved in the modulation of inflammation after myocardial injury that to our knowledge has not been investigated before. We demonstrate that inhibition of the interaction between PDPN-expressing cells and PDPN-binding cells reduces but does not fully suppress the inflammation after MI, and at the same time, enhances an endogenous myocardial regeneration process after ischemic injury.

PDPN plays a pivotal role in heart formation and is necessary for epicardial development and myocardial differentiation (22). PDPN-null mice have increased embryonic lethality, impairment in development of the proepicardial organ, epicardial adhesion, and spreading and migration of epicardium-derived cells (22). The epicardial pathology is correlated with reduced epithelial-mesenchymal transformation caused by upregulation of E-cadherin, a protein normally downregulated by PDPN (22). In the adult heart, PDPN at baseline is expressed only by lymphatic endothelial cells and importantly (15), reappears in the infarcted murine hearts as early as 2 days after MI where it is widely expressed by a heterogeneous cohort of cells with a variable potency to adopt the lymphatic endothelial or mesenchymal fates (3). Why PDPN reappears in the heart only during the inflammatory phases is unknown. We demonstrated that PDPN is a marker of injured myocardium; the fibrotic areas of hearts from animals treated with Ang II are positive for PDPN in immunostaining; moreover, we showed that PDPN is expressed in the fibrotic tissue of ischemic human hearts. Similar to the ischemic heart, PDPN is upregulated in human oral and mouse skin carcinomas where E-cadherin is downregulated by PDPN, resulting in upregulation of epithelial-mesenchymal transformation leading to invasive growth and metastasis of the carcinoma cells (19). It has been shown recently that PDPN increases in several different pathologies $(9,10)$.

PDPN binds its receptor CLEC-2, which is highly expressed by platelets and a variety of immune cells $(4,19)$; thus, PDPN is involved in inflammation and may represent a key mediator of cross-talk between progenitor or mesenchymal resident cells and inflammatory cells recruited from the circulation at the site of injury. In concordance with a previous report (3), we confirmed that PDPN is not expressed in the heart by inflammatory cells, and resident PDPN-positive cells in the heart do not come from the circulation. Only $20 \%$ of PDPN-positive cells in infarcted hearts express hematopoietic markers such as F4/80, Ly6C, and CD11b. For this reason, the PDPN-expressing pools do not correspond to maturing macrophages because only a small population of circulating monocytes can acquire PDPN during inflammation (7). To address the role of PDPN in wound healing and scar structure, we blocked its activity using a neutralizing $\mathrm{Ab}$ (Supplemental Figure 6). Because PDPN-knockout mice do not survive the embryonic phase, they do not represent an appropriate model for our study (22). Therapeutic approaches using PDPN-neutralizing Ab have been shown to suppress tumor metastasis through Ab-dependent cellular cytotoxicity (31) or control of cellular invadopodia stability (32). Podoplanin is required for the complete maturation and stabilization of invadopodia (32). In our model, we did not observe any differences in PDPN expression in acute and chronic phases after MI between the treated and untreated groups. Treatment with the neutralizing Ab does not affect the cell migration or the cell composition in the scar; thus, PDPN-expressing cells resident in the heart displayed PDPN under inflammatory conditions, and the neutralizing Ab was only able to interfere in the interaction of PDPN with PDPN-binding cells. Similar observations were reported by other groups studying the biology of inflammation and possible selective targets in rheumatoid arthritis (11), psoriasis (10), sepsis (12), and deep vein thrombosis (13).

Activation of inflammatory cascades in the infarcted heart stimulates a wide range of cellular responses (1). During the granulation phase, neutrophils and monocytes clear the dead cells from the wound, and consequently, antiinflammatory stimuli promote repair enhancing the recruitment of progenitor cells involved in angiogenesis after MI and cardiac scar formation (30). Based on the tissue damage, the proinflammatory signaling could be prolonged and accentuate adverse remodeling by activating proteases, transducing proapoptotic cascades in cardiomyocytes, and promoting matrix degradation (26). Our results showed that PDPN neutralization reduced the scar size, suggesting possible inhibition of cell death during the inflammatory phase and supporting the repair during the proliferative and maturation phases. In fact, as early as 3 days after MI, animals treated with PDPN-neutralizing Ab displayed a reduced cell death probably due to the attenuated proinflammatory response. The massive cell death after ischemia results also in poorly vascularized scars; the consequent low blood flow inhibits the cell differentiation leading to superseding repair and fibrosis (33). Mature scars of animals injected with PDPN-neutralizing Ab after 
MI showed an enhanced vascularization, suggesting a potentially fertile microenvironment for progenitor cell differentiation. Moreover, during the proliferative phase, activation of endogenous fibrogenic signals stimulate and exacerbate extracellular matrix deposition in the ischemic area and alteration in the tissue structure, and cardiac morphology results in severe left ventricle dysfunction (26). In our model, we observe an organized fibronectin deposition in structures similar to swirls; this type of structure is usually observed during new vessel formation. On the contrary, control animals show massive fibronectin assemblies along the ischemic area. Of note, PDPN has been described to be important for lymphatic vessel spreading, and the new lymphangiogenesis is essential for the reduction of edema and fibrosis following MI (34). Animals treated with neutralizing $\mathrm{Ab}$ do not express a reduction in new lymphangiogenesis 30 days after MI, suggesting that lymphatic endothelial cells do not represent an off-target effect.

Cardiomyocyte regeneration after MI remains controversial and the degree of regeneration remains hotly debated (35). New myocytes rarely differentiate from circulating or resident progenitor cells (36). Previous reports have suggested that a healthy scar environment with pronounced neovascularization, reduced ROS, and correct alignment of the scar fibers may support the engraftment, proliferation, and differentiation of progenitor cells (33). Cardiac progenitor cells recruited to the injured heart are usually characterized by the expression of NKX-2.5, a myocyte transcription factor expressed at the early stage of progenitor/stem cell differentiation and which represents an important marker for myocyte fate (33). We observed NKX-2.5-positive cell assemblies in the ischemic area of animals treated with PDPN-neutralizing Ab on day 7 after MI; the pool of NKX-2.5-positive cells often coexpressed troponin T. The presence of undifferentiated cells with myocyte lineage at an early time point after injury correlated with the presence of bands of differentiated small myocytes in treated animals at 30 days after MI. In the absence of lineage-tracing experiments, we could not positively link small myocytes in the scar area as a direct descendent of NKX-2.5-positive progenitors; however, these data suggest that PDPN neutralization may exert beneficial effects during the granulation and proliferation phases stimulating the recruitment and engraftment of putative NKX-2.5-positive cardiac progenitors.

Targeting a select pathway during the inflammation phase that suppresses injurious processes without interfering with the quality of reparative response is reflected not only on the size and geometry of the scar itself but also on heart function (2). Animals treated with PDPN-neutralizing Ab perform a better contractility, and the observed small myocyte islets resident in the scar tissue may contribute to the LV function and contraction. The histological data, in relation to the functional data, demonstrated that the neutralizing activity of PDPN leads to the healthier tissue geometry organization in the treated animals compared with scar formation in the control animals. Based on the sequential recruitment of inflammatory cells in the proinflammatory, proliferative, and reparative phases after MI (27), we focused our attention on the monocyte populations as a priority over other described PDPN-binding cells (21). Coculture of activated monocytes with PDPN-positive cells stimulated monocytes to produce proinflammatory cytokines, which are important for the recruitment of other immune cells, and that blocking of the interaction between the monocytes and PDPN-positive cells reduces the proinflammatory cytokine expression while simultaneously enhancing the expression of antiinflammatory cytokines such as IL-10 and reduced expression of CD68 ${ }^{+}$cells. These observations open the possibility that therapeutic benefits of PDPN neutralization may in part depend on the inhibition of monocyte interaction with PDPN-positive cells leading to the upregulation of antiinflammatory cytokines and to potentially a favorable microenvironment in the treated animals for the granulation phase and subsequent repair. In vivo treatment with PDPN-neutralizing Ab led to increased presence of reparative $\mathrm{CD} 163^{+}$monocytes/macrophages. The total number of monocytes/macrophages $\left(\mathrm{CD} 86^{+}\right)$recruited in the hearts 3 days after MI was similar in both groups of animals, but the percentage of cells double positive for CD86/CD206 monocytes/macrophages was much higher in the treated animals. Additionally, the cardiac expression of antiinflammatory cytokine IL-10 was also significantly elevated. It has been reported that IL-10 inhibits inflammation in the heart (37) and enhances myocardial repair and function after MI (37); specifically, IL-10 attenuates LV dysfunction and remodeling with effects on fibrosis and capillary density $(38,39)$. Interestingly, PDGFR- $\beta$ and VEGF-A are upregulated in treated animals; these results may explain observed differences in angiogenesis that we detected in histological analysis.

One major finding of the present study is that PDPN neutralization leads to a balance of proinflammatory and antiinflammatory signals; this equilibrium may preserve the heart from exacerbated ischemic injury and stimulate a healthy scar formation. In past decades, several clinical trials using potent antiinflammatory drugs or neutralizing Abs for adhesion molecules, cytokines, and chemokines have been used as a therapy for MI; unfortunately, none of them showed the expected outcomes (2). These studies suggested 
that dynamic alteration in cytokines and growth factor expression in the heart impaired the differentiation of several distinct subpopulations of macrophages in the inflammatory and reparative response (27). Proinflammatory cytokines are necessary for the recruitment of phagocytic cells in the inflammatory phase, but at the same time, a proreparative and proregenerative microenvironment is essential for the tissue survival and the inhibition of catastrophic myocardial remodeling (27). Our findings on PDPN neutralization may potentially open a new perspective in the inflammatory balance after MI and PDPN interactome may potentially represent a novel target for cardioprotective therapies.

\section{Methods}

Animals and surgeries. Eight to 10-week-old male WT (C57BL/6J) and 8- to 10-week-old male global GFP mice (C57BL/6-Tg [UBC-GFP] 30Scha/J) were purchased from Jackson Research Laboratory. Before treatments and at 7 and 30 days after MI, all animals were screened for baseline and post-MI echocardiography. MI was induced through LV descending coronary artery ligation as previously described (3). Following MI, animals were injected i.p. with $25 \mu \mathrm{g}$ of PDPN-neutralizing Ab (Santa Cruz, sc-134483), $25 \mu \mathrm{g}$ of rabbit IgG control Ab (Cell Signaling Technology, 2729S), or $250 \mu 1$ of saline solution per animal at 1, 2, 7, and 15 days after MI. At 2, 3, 7 and 30 days after MI, animals were sacrificed under deep anesthesia, bilateral thoracotomy was performed, the hearts were removed and either fixed and processed for histological analysis, or enzymatically digested (40) for single-cell assessment by flow cytometry or cell culture purpose, as described in the information to follow. LV hypertrophy and heart failure was induced in 8- to 10-weekold male WT (C57BL/6J) mice with Ang II (1.6 mg/kg/d for 28 days) infusion using mini-osmotic pumps as described previously (41). Twenty-eight days after treatment, mice were euthanized after final functional assessments and LV tissue samples were collected for histology analysis.

Transthoracic echocardiographic analysis. Echocardiography was performed using the Vevo 2100 imaging system from VisualSonics, as published $(37,38)$. Briefly, transthoracic 2-dimensional echocardiography in mice anesthetized with $1 \%$ to $3 \%$ isoflurane was performed with the probe. M-mode echocardiography was carried out in the parasternal short axis in mice to assess heart rate, the LV internal diameter in diastole and systole, and the LV anterior and posterior wall in diastole and systole. LV FS percentage and EF percentage were calculated (42).

In vivo hemodynamic measurements. In vivo cardiac hemodynamic measurements were performed as described $(37,38)$. Mice were anesthetized with $2 \%$ tribromoethanol (Sigma), and the right common carotid artery was isolated and cannulated with a 1.4-F micromanometer (Millar Instruments). LV pressure and heart rate were measured by this catheter advanced into the LV cavity, and data were recorded and analyzed on a PowerLab System (AD Instruments Pty Ltd.). These parameters as well as maximal values of the instantaneous first derivative of $\mathrm{LV}$ pressure $\left(+\mathrm{dP} / \mathrm{dt}_{\max }\right.$, as a measure of cardiac contractility) and minimum values of the instantaneous first derivative of $\mathrm{LV}$ pressure $\left(-\mathrm{dP} / \mathrm{dt}_{\min }\right.$, as a measure of cardiac relaxation) were recorded at baseline and after administration of the $\beta$-adrenergic receptor agonist, isoproterenol $(0.1,0.5,1,5$, and $10 \mathrm{ng})(43)$.

Human heart tissue samples. Heart tissue samples $(n=5)$ were obtained from patients with ischemic cardiomyopathy at the time of transplantation at the Temple University Cardiovascular Research Center and immediately frozen in liquid nitrogen and stored at $-80^{\circ} \mathrm{C}$ until use. Tissue collection was conducted in accordance with the Declaration of Helsinki and approved by the Temple University Institutional Review Board.

Immunohistochemistry of thin cardiac sections. Mouse hearts were washed with PBS, 10\% formalin fixed at least for 48 hours with $10 \%$ formalin, and embedded in paraffin. Human heart tissue samples were fixed in $10 \%$ formalin and embedded in paraffin. Cardiac tissues were cut into 5 - to $4-\mu$ m-thick sections. Following deparaffinization and rehydration, mouse and human samples were stained with Masson's trichrome staining (Sigma-Aldrich, HT1079-1set and HT15-1kt) and after heat-induced antigen retrieval ( $\mathrm{pH}$ 6.0), indirectly immunolabeled with commercially available primary antibodies (Supplemental Table 1) and corresponding fluorophore-conjugated secondary reagents (Supplemental Table 1) or directly stained for TUNEL in red (Roche, 12156792910). Nuclei were counterstained with DAPI (Sigma-Aldrich). Multiple sections from the hearts of at least 3 mice for each time point after MI were examined, and representative time points are described in the figure legends. Images were acquired with Niko Eclipse Ti fluorescence microscope using $\times 4, \times 10, \times 20, \times 40, \times 60$, and $\times 100$ objectives. Optical sections were projected into a single plane for each color channel and merged using Adobe Photoshop (Adobe) software. Quantitative image analysis was performed with NIH ImageJ by scoring multiple imaging fields. 
Isolation and culture of PDPN-positive cells. WT and global GFP mice were euthanized at 2 days after induction of MI, as described previously. The hearts were excised and extensively washed in PBS. The cardiac tissues were minced and subjected to repetitive rounds of enzymatic digestion with collagenase type 2 (Worthington Biochemical Corp., LS004176) until complete dissociation. Larger cells, such as mature myocytes, were precipitated, and the supernatants containing small cell populations were filtered through $40-\mu \mathrm{m}$ cell strainers. For positive cell separation (magnetic column 130-042-401), the small cell population was incubated with PDPN Ab (R\&D Systems, BAF3244) previously bonded (overnight at $4^{\circ} \mathrm{C}$ ) with magnetic beads (anti-biotin MicroBeads, Miltenyi Biotec, 130-090-485) for 20 minutes at $4^{\circ} \mathrm{C}$. After washing, the cells were cultured in expansion medium (EBM-2 medium [CC-4176], supplemented with CC-4176 [both from Lonza] and Endothelial Cell Growth Supplement [Bio-Rad 4110-5004, without antibiotics] on collagen-coated flasks [Sigma-Aldrich, C8919]). The purity of the isolation was analyzed by flow cytometry for PDPN, LYVE-1, and CD68 on detached (0.2\% trypsin) and fixed (4\% paraformaldehyde [PFA]) cultured PDPN-positive cells.

PDPN neutralization activity. PDPN-positive cells with and without PDPN-neutralizing Ab $(5 \mu \mathrm{g} / \mathrm{mL}$ of pAb anti-PDPN) were seeded on the inner side of transwell insets (Corning, $0.8 \mu \mathrm{m}$ ) and allowed to migrate from the basal to the apical side of transwell inserts in different conditions (with and without LPS) for 24 hours. PDPN-positive cells migrated to the apical side were detached with $0.2 \%$ trypsin and the absolute number of migrating cells was counted by flow cytometry.

Isolation and monocyte culture. BM monocytes were isolated from BM mononuclear cells of mice fore and hindlimbs with density-gradient centrifugation as described previously (25). Mononuclear cells were seeded on plastic dishes and the medium changed after 40 minutes. The monocyte population was cultured in DMEM with $20 \%$ FBS, $1 \%$ penicillin-streptomycin solution, and $20 \%$ of L929-conditioned medium. Monocytes were activated with $100 \mathrm{ng} / \mathrm{mL}$ of LPS for 24 hours prior to coculture with PDPN-positive cells. Flow cytometry analysis of activated monocytes was performed on detached (TrypLE Express, Gibco, 12604-021) and fixed (4\% PFA) cells for CD68, CD86, CD163, and CD206.

Isolation of peripheral blood mononuclear cells. Peripheral blood from control (sham-operated) animals and animals at 1 and 2 days after MI was collected in heparin-loaded syringes after carotid artery incision under deep anesthesia. Single-cell suspensions were fixed with 4\% PFA, and flow cytometry analysis was performed for PDPN and BM lineage markers.

Coculture of PDPN-positive cells and activated monocytes. PDPN-positive cells were cocultured for 24 hours with activated monocytes in the presence or absence of PDPN-neutralizing Ab ( $5 \mu \mathrm{g} / \mathrm{mL}$ of pAb anti-PDPN, R\&D Systems, AF3244). Prior to coculture, PDPN-positive cells were pretreated with neutralizing Ab for 8 hours. Conditioned medium from PDPN cells only, PDPN cells treated with neutralizing Ab, PDPN cells with activated monocytes, and PDPN cells pretreated with PDPN-neutralizing Ab and cocultured with activated monocytes were analyzed by ELISA for the major proinflammatory and antiinflammatory cytokines. Multiplex ELISA was performed by a third party (Evetechnologies: https://www.evetechnologies.com). After coculture, PDPN-positive cells with activated monocytes and PDPN cells pretreated with PDPN-neutralizing $\mathrm{Ab}$ and cocultured with activated monocytes were detached with $0.2 \%$ trypsin and a scraper and either fixed with 4\% PFA and stained with CD68 and CD163 and analyzed by flow cytometry or separated for positive cell separation (magnetic column). The cells were incubated with anti-GFP Ab (Invitrogen, A10259) previously bonded (overnight at $4^{\circ} \mathrm{C}$ ) with magnetic beads (anti-biotin MicroBeads, Miltenyi Biotec, 130-090-485) for 20 minutes at $4^{\circ} \mathrm{C}$. After sorting, the cells were lysed for RNA extraction.

ELISA. Mouse cytokine 32-plex discovery assay was performed by Eve Technologies (42) on cell culture supernatant of PDPN cells only, PDPN cells treated with neutralizing Ab, PDPN cells with activated monocytes, and PDPN cells pretreated with PDPN-neutralizing Ab and cocultured with activated monocytes. Cytokine profile was determined using a 32-plex luminex-based platform and the analysis was performed on 3 different samples for each condition.

$q R T-P C R$. Expression levels of different genes were measured using qRT-PCR. Total cellular RNA was isolated from mouse myocardial tissue at 3 days after MI of animals treated and untreated with PDPN-neutralizing $\mathrm{Ab}$, or from monocytes separated from the PDPN-positive cells after coculture with and without the presence of PDPN-neutralizing Ab using miRNeasy Mini Kit (Qiagen) following the manufacturer's instructions (44). The cDNA was obtained from total RNA using the High-Capacity cDNA Reverse Transcription Kit (Applied Biosystems, 4368814). Real-time PCR was performed on an Applied Biosystems 770 apparatus. The list of genes with the corresponding primers is shown in Supplemental Table 2. 
Flow cytometry analysis of isolated and cultured cardiac cells. Infarcted WT mice treated and untreated (saline solution injected) with PDPN- neutralizing Ab were euthanized 3 days after MI as described above. The hearts were excised and extensively washed in PBS. The cardiac tissues were minced and subjected to repetitive rounds of enzymatic digestion with collagenase type 2 (Worthington Biochemical Corp.) until complete dissociation. Larger cells, such as mature myocytes, were precipitated, and the supernatants containing small cell populations were filtered through $70-\mu \mathrm{m}$ cell strainers. The small cell population was fixed with $4 \%$ PFA and stained for CD86, CD68, CD163, and CD206. Nonimmune normal donkey and rat IgGs and isotype controls were employed as negative controls for the respective antigen-specific labeling. Samples were acquired with and analyzed using an LSR-II flow cytometer. Single cells were gated using FSC-A/SSC-A followed by FSC-H/FSC-W and SSC-H/SSC-W in all experiments. Compensation settings, gating of positive populations and calculations of the percentage of positive cells were performed based on nonimmune and isotype IgGs and fluorescence.

Statistics. Statistical analysis was performed with the 2-tailed $t$ test for single comparison within 2 groups or with 1-way or 2-way ANOVA for multiple comparisons using GraphPad Prism. Data are expressed as mean \pm SEM. Bonferroni's post hoc test was used among all groups when applicable, and a value of $P<$ 0.05 was considered statistically significant.

Study approval. All animal experiments were conducted according to the NIH Guide for the Care and Use of Laboratory Animals (National Academies Press, 2011) and were approved by the Institutional Animal Care and Use Committee of Temple University. Human cardiac tissue collection was conducted in accordance with the Declaration of Helsinki and approved by the Temple University Institutional Review Board.

\section{Author contributions}

MC was responsible for conception and design, collection and/or assembly of data, data analysis and interpretation, and manuscript writing. VNSG, ZC, CL, DAG, AML, MMT, RR, CW, and CB collected and/or assembled data. WJK was responsible for data interpretation and design. RK was responsible for conception and design, final editing, and approval of the manuscript.

\section{Acknowledgments}

This work was funded in part by NIH grant HL091983, and NIH grants HL126186 and HL134608 (to RK).

Address correspondence to: Raj Kishore, Center for Translational Medicine, Temple University School of Medicine, MERB-953, 3500 North Broad Street, Philadelphia, Pennsylvania 19140, USA. Phone: 215.707.2523; Email: raj.kishore@temple.edu.

1. Frangogiannis NG. The immune system and the remodeling infarcted heart: cell biological insights and therapeutic opportunities. J Cardiovasc Pharmacol. 2014;63(3):185-195.

2. Saxena A, Russo I, Frangogiannis NG. Inflammation as a therapeutic target in myocardial infarction: learning from past failures to meet future challenges. Transl Res. 2016;167(1):152-166.

3. Cimini M, Cannatá A, Pasquinelli G, Rota M, Goichberg P. Phenotypically heterogeneous podoplanin-expressing cell populations are associated with the lymphatic vessel growth and fibrogenic responses in the acutely and chronically infarcted myocardium. PLoS ONE. 2017;12(3):e0173927.

4. Astarita JL, Acton SE, Turley SJ. Podoplanin: emerging functions in development, the immune system, and cancer. Front Immunol. 2012;3:283.

5. Quintanilla M, Montero-Montero L, Renart J, Martín-Villar E. Podoplanin in inflammation and cancer. Int J Mol Sci. 2019;20(3):E707.

6. Pan Y, Xia L. Emerging roles of in vascular development and homeostasis. Front Med. 2015;9(4):421-430.

7. Ugorski M, Dziegiel P, Suchanski J. Podoplanin - a small glycoprotein with many faces. Am J Cancer Res. 2016;6(2):370-386.

8. Braun N, et al. The spectrum of podoplanin expression in encapsulating peritoneal sclerosis. PLoS ONE. 2012;7(12):e53382.

9. Nazari B, et al. Altered dermal fibroblasts in systemic sclerosis display podoplanin and CD90. Am J Pathol. 2016;186(10):2650-2664

10. Noack M, Ndongo-Thiam N, Miossec P. Role of podoplanin in the high interleukin-17A secretion resulting from interactions between activated lymphocytes and psoriatic skin-derived mesenchymal cells. Clin Exp Immunol. 2016;186(1):64-74.

11. Noack M, Ndongo-Thiam N, Miossec P. Interaction among activated lymphocytes and mesenchymal cells through podoplanin is critical for a high IL-17 secretion. Arthritis Res Ther. 2016;18:148.

12. Rayes J, et al. The podoplanin-CLEC-2 axis inhibits inflammation in sepsis. Nat Commun. 2017;8(1):2239.

13. Payne H, Ponomaryov T, Watson SP, Brill A. Mice with a deficiency in CLEC-2 are protected against deep vein thrombosis. Blood. 2017;129(14):2013-2020.

14. Näpänkangas J, et al. Increased mesenchymal podoplanin expression is associated with calcification in aortic valves. Cardiovasc Pathol. 2019;39:30-37. 
15. Pinto AR, et al. Revisiting cardiac cellular composition. Circ Res. 2016;118(3):400-409.

16. Brakenhielm E, Alitalo K. Cardiac lymphatics in health and disease. Nat Rev Cardiol. 2019;16(1):56-68.

17. Loukas M, Abel N, Tubbs RS, Grabska J, Birungi J, Anderson RH. The cardiac lymphatic system. Clin Anat. 2011;24(6):684-691.

18. Acton SE, et al. Podoplanin-rich stromal networks induce dendritic cell motility via activation of the C-type lectin receptor CLEC-2. Immunity. 2012;37(2):276-289.

19. Suzuki-Inoue K, Osada M, Ozaki Y. Physiologic and pathophysiologic roles of interaction between C-type lectin-like receptor 2 and podoplanin: partners from in utero to adulthood. J Thromb Haemost. 2017;15(2):219-229.

20. Navarro-Núñez L, Langan SA, Nash GB, Watson SP. The physiological and pathophysiological roles of platelet CLEC-2. Thromb Haemost. 2013;109(6):991-998.

21. Lowe KL, et al. The expression of mouse CLEC-2 on leucocyte subsets varies according to their anatomical location and inflammatory state. Eur J Immunol. 2015;45(9):2484-2493.

22. Mahtab EA, et al. Cardiac malformations and myocardial abnormalities in podoplanin knockout mouse embryos: correlation with abnormal epicardial development. Dev Dyn. 2008;237(3):847-857.

23. Kaneko MK, et al. Chimeric anti-podoplanin antibody suppresses tumor metastasis through neutralization and antibody-dependent cellular cytotoxicity. Cancer Sci. 2012;103(11):1913-1919.

24. Kim JH, Jiang YP, Cohen IS, Lin RZ, Mathias RT. Pressure-overload-induced angiotensin-mediated early remodeling in mouse heart. PLoS ONE. 2017;12(5):e0176713.

25. Verma SK, et al. Interleukin-10 inhibits bone marrow fibroblast progenitor cell-mediated cardiac fibrosis in pressure-overloaded myocardium. Circulation. 2017;136(10):940-953.

26. Rog-Zielinska EA, Norris RA, Kohl P, Markwald R. The living scar—cardiac fibroblasts and the injured heart. Trends Mol Med. 2016;22(2):99-114.

27. Frangogiannis NG. Emerging roles for macrophages in cardiac injury: cytoprotection, repair, and regeneration. J Clin Invest. 2015;125(8):2927-2930.

28. Mourão-Sá D, et al. CLEC-2 signaling via Syk in myeloid cells can regulate inflammatory responses. Eur J Immunol. 2011;41(10):3040-3053.

29. Mosser DM, Zhang X. Interleukin-10: new perspectives on an old cytokine. Immunol Rev. 2008;226:205-218.

30. Prabhu SD, Frangogiannis NG. The biological basis for cardiac repair after myocardial infarction: from inflammation to fibrosis. Circ Res. 2016;119(1):91-112.

31. Kunita A, Kaneko MK, Ogasawara S, Fukayama M, Kato Y. Chimeric anti-podoplanin mAbs produced by CasMab technology possess high antitumor activity via ADCC and CDC activities. Eur J Cancer. 2016;69(supp1 1):S57.

32. Martín-Villar E, et al. Podoplanin mediates ECM degradation by squamous carcinoma cells through control of invadopodia stability. Oncogene. 2015;34(34):4531-4544.

33. Furtado MB, Nim HT, Boyd SE, Rosenthal NA. View from the heart: cardiac fibroblasts in development, scarring and regeneration. Development. 2016;143(3):387-397.

34. Henri O, et al. Selective Stimulation of Cardiac Lymphangiogenesis Reduces Myocardial Edema and Fibrosis Leading to Improved Cardiac Function Following Myocardial Infarction. Circulation. 2016;133(15):1484-1497.

35. van Berlo JH, et al. c-kit ${ }^{+}$cells minimally contribute cardiomyocytes to the heart. Nature. 2014;509(7500):337-341.

36. Vicinanza C, et al. Kit ${ }^{\text {cre }}$ knock-in mice fail to fate-map cardiac stem cells. Nature. 2018;555(7697):E1-E5.

37. Krishnamurthy P, Rajasingh J, Lambers E, Qin G, Losordo DW, Kishore R. IL-10 inhibits inflammation and attenuates left ventricular remodeling after myocardial infarction via activation of STAT3 and suppression of HuR. Circ Res. 2009;104(2):e9-18.

38. Krishnamurthy $\mathrm{P}$, et al. Interleukin-10 deficiency impairs bone marrow-derived endothelial progenitor cell survival and function in ischemic myocardium. Circ Res. 2011;109(11):1280-1289.

39. Robertson TE, Nikolic-Paterson DJ, Hurst LA, Atkins RC, Chadban SJ. IL-10 induces mesangial cell proliferation via a PDGF-dependent mechanism. Clin Exp Immunol. 2002;130(2):241-244.

40. Cavasin MA, Tao Z, Menon S, Yang XP. Gender differences in cardiac function during early remodeling after acute myocardial infarction in mice. Life Sci. 2004;75(18):2181-2192.

41. Kishore R, et al. Interleukin-10 inhibits chronic angiotensin II-induced pathological autophagy. J Mol Cell Cardiol. 2015;89(Pt B):203-213.

42. Garikipati VNS, et al. Therapeutic inhibition of miR-375 attenuates post-myocardial infarction inflammatory response and left ventricular dysfunction via PDK-1-AKT signalling axis. Cardiovasc Res. 2017;113(8):938-949.

43. Gao E, et al. A novel and efficient model of coronary artery ligation and myocardial infarction in the mouse. Circ Res. 2010;107(12):1445-1453.

44. Borghetti G, et al. Notch signaling modulates the electrical behavior of cardiomyocytes. Am J Physiol Heart Circ Physiol. 2018;314(1):H68-H81.

45. Wells KJ, et al. Assessing needs and assets for building a regional network infrastructure to reduce cancer related health disparities. Eval Program Plann. 2014;44:14-25. 